\title{
Early Experience with Two Techniques of Intrascleral Intraocular Lens Fixation
}

\author{
Tomoyuki Kunishige and Hiroshi Takahashi
}

\begin{abstract}
Department of Ophthalmology, Nippon Medical School, Tokyo, Japan
Background: We compared our early experience of visual outcomes, operation time, and complications for two techniques of intrascleral intraocular lens (IOL) fixation: T-fixation and flanged IOL fixation.

Methods: Data from patients who underwent scleral fixation of an IOL between October 2017 and December 2018 were analyzed retrospectively. Intraoperative time for fixation steps, corneal endothelial cell density (ECD) rate reduction, and intraoperative/postoperative complications were compared between T-fixation (Group T, n =4) and flanged IOL fixation (Group F, n =6).

Results: Mean patient age was $73.7 \pm 13.0$ years. Intraoperative time was significantly longer for Group $\mathrm{T}(27.5 \pm 9.7 \mathrm{~min})$ than for Group F $(13.0 \pm 3.8 \mathrm{~min}$; P=0.03, Mann-Whitney U-test). Intraoperative complications included iris damage from the $30-\mathrm{G}$ needle in 2 eyes in Group F. Postoperative complications included iris capture by the IOL in 1 eye in Group F. No incidents of postoperative retinal detachment or IOL dislocation were identified. The ECD reduction rate did not significantly differ between groups. Conclusions: Both techniques yielded favorable surgical outcomes. Flanged IOL fixation might be superior because of its shorter intraoperative time but could initially be technically difficult because of the need to perform angled sclerotomy with a 30-G needle to avoid iris injury.
\end{abstract}

(J Nippon Med Sch 2020; 87: 138-141)

Key words: intrascleral intraocular lens fixation techniques, T-fixation technique, flanged IOL fixation

\section{Introduction}

Secondary intraocular lens (IOL) implantation is an essential technique for compromised capsular bag and zonules. Transscleral IOL suture was the most widely used technique because of its long-term safety and effectiveness ${ }^{1-4}$. However, this technique sometimes causes 100 polypropylene suture erosion, breakage, and delayed IOL dislocation. A new technique that addresses these drawbacks, intrascleral fixation, has been described as a sutureless technique for IOL fixation ${ }^{5,6}$. Various techniques for scleral-fixated IOL implantation have been described $^{5-13}$. Each has its own learning curve during early cases. Ohta et al. described T-fixation as a new intrascleral fixation technique that does not require large lamellar scleral flaps or fibrin glue ${ }^{8,9}$. Yamane et al. developed flanged IOL fixation as a new surgical procedure that can be carried out via the conjunctiva in which the haptics of the IOL are fixed securely to the sclera, without using suture or glue $\mathrm{e}^{10,11}$.
This retrospective study compared visual outcomes and complications for early cases after treatment with Tfixation and flanged IOL fixation.

\section{Materials and Methods \\ Patients and Methods}

This study adhered to the tenets of the Declaration of Helsinki and was approved by the Institutional Review Board and Ethics Committee of Nippon Medical School (No. R1-06-1155). We followed the retrospective observational research information disclosure procedure (opt-out) of Nippon Medical School when obtaining informed consent from research participants. A retrospective review of consecutive patients who underwent scleral fixation of IOL was undertaken by using medical records and retained images.

The medical records of consecutive patients who underwent scleral fixation of IOL at Nippon Medical School between October 2017 and December 2018 were exam-

Correspondence to Tomoyuki Kunishige, Department of Ophthalmology, Nippon Medical School, 1-1-5 Sendagi, Bunkyo-ku, Tokyo 113-8603, Japan

E-mail: s9038@nms.ac.jp

https://doi.org/10.1272/jnms.JNMS.2020_87-304

Journal Website (https://www.nms.ac.jp/sh/jnms/) 
Group T

Group F

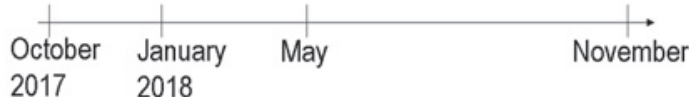

Fig. 1 Date of scleral-fixated IOL implantation.

ined retrospectively. The same surgeon (T.K.) performed all scleral fixation of IOLs early in his experience with the techniques. Intraoperative time for the IOL fixation steps and intraoperative/postoperative complications were compared between T-fixation (Group T, n $=4$ ) and flanged IOL fixation (Group F, n = 6). Corneal endothelial cell density (ECD) was assessed by non-contact specular microscopy (Noncon Robo; Konan, Hyogo, Japan) at the center of the cornea. ECD reduction rate was calculated as the difference between preoperative ECD and ECD at 3 months.

\section{Surgical Procedures}

In Group $\mathrm{T}$, conjunctival peritomy was done at the 2 o'clock and 8 o'clock positions under retrobulbar anesthesia. Two T-shaped incisions were made, $2.0 \mathrm{~mm}$ from the limbus, exactly $180^{\circ}$ apart diagonally, and an infusion cannula or anterior chamber maintainer was inserted. Sclerotomy was performed parallel to the iris at the Tshaped incision with a 25-gauge knife, and a scleral tunnel was made parallel to the limbus at the branch point of the T-shaped incision. A 3.0-mm sclerocorneal tunnel incision was created at the 10 to 12 o'clock position, through which a 3-piece IOL (NX-70 Santen Pharmaceutical Co., Osaka, Japan) was implanted with an injector. The tip of the haptic was then grasped with 25-G forceps and pulled through the sclerotomy. After the trailing haptic was inserted into the anterior chamber, the haptic tip was grasped with 25-G forceps and pulled through the other sclerotomy. The haptic was subsequently inserted into the limbus-parallel scleral tunnel. The sclera tunnel and conjunctival peritomy were sutured with 10-0 Nylon or 8-0 Vicryl.

The technique required to make a sclerotomy with a 25-G knife and use forceps to externalize the IOL haptic is relatively easy. However, substantial time is required to dissect the conjunctiva, make the scleral tunnel, insert the haptics into the scleral tunnel, and suture the conjunctival peritomy.

In Group F, an infusion cannula or anterior chamber maintainer was inserted under retrobulbar anesthesia. An
Table 1 Demographic characteristics

\begin{tabular}{lcc}
\hline & Group T & Group F \\
\hline Mean Age \pm SD & $78.0 \pm 5.9$ & $73.7 \pm 13.0$ \\
Male & 1 & 6 \\
Female & 3 & 0 \\
\hline
\end{tabular}

Table 2 Indications for surgery

\begin{tabular}{lcc}
\hline & Group T & Group F \\
\hline Dislocated IOL & 1 & 1 \\
Subluxated cataract & 1 & 2 \\
Aphakia & 2 & 3 \\
\hline
\end{tabular}

angled sclerotomy was made through the conjunctiva with a 30-G thin-wall needle (TSK ultra-thin-wall needle; Tochigi Seiko, Tochigi, Japan) at $2 \mathrm{~mm}$ from the limbs. A 3.0-mm sclerocorneal tunnel incision was created at the 12 to 2 o'clock position, through which a 3-piece IOL (NX-70 Santen Pharmaceutical Co., Osaka, Japan) was implanted with an injector. The leading haptic was threaded into the lumen of the needle by using forceps. The trailing haptic was inserted into the lumen of the second needle. Both haptics were externalized onto the conjunctiva by using the flanged IOL fixation. The ends of the haptics were cauterized with an ophthalmic cautery device (Accu-Temp Cautery; Beaver Visitec, Waltham, MA) to make a flange. The flange of the haptics was pushed back and fixed into the scleral tunnels.

A 30-G needle was used to externalize the haptics of the IOL. The technique without a scleral flap is simpler and requires no sutures. However, making an angled sclerotomy through the conjunctiva with a $30-\mathrm{G}$ needle, to avoid iris injury, and introducing the haptic into the 30-G needle in the anterior chamber is difficult.

\section{Statistical Analysis}

The ECD reduction rate and intraoperative time for intrascleral IOL fixation techniques in both groups were compared with the Mann-Whitney U-test. A $P$ value of $<0.05$ was considered to indicate statistical significance.

\section{Results}

Data were analyzed from 10 eyes of 10 consecutive patients: intrascleral IOL fixation using T-fixation technique in 4 eyes (Group T) and flanged IOL fixation in 6 eyes (Group F) (Fig. 1). Patient age was 53 to 87 years. Group $\mathrm{T}$ comprised 1 man and 3 women and Group F comprised 6 men (Table 1).

Table 2 shows the underlying pathologies: dislocated 

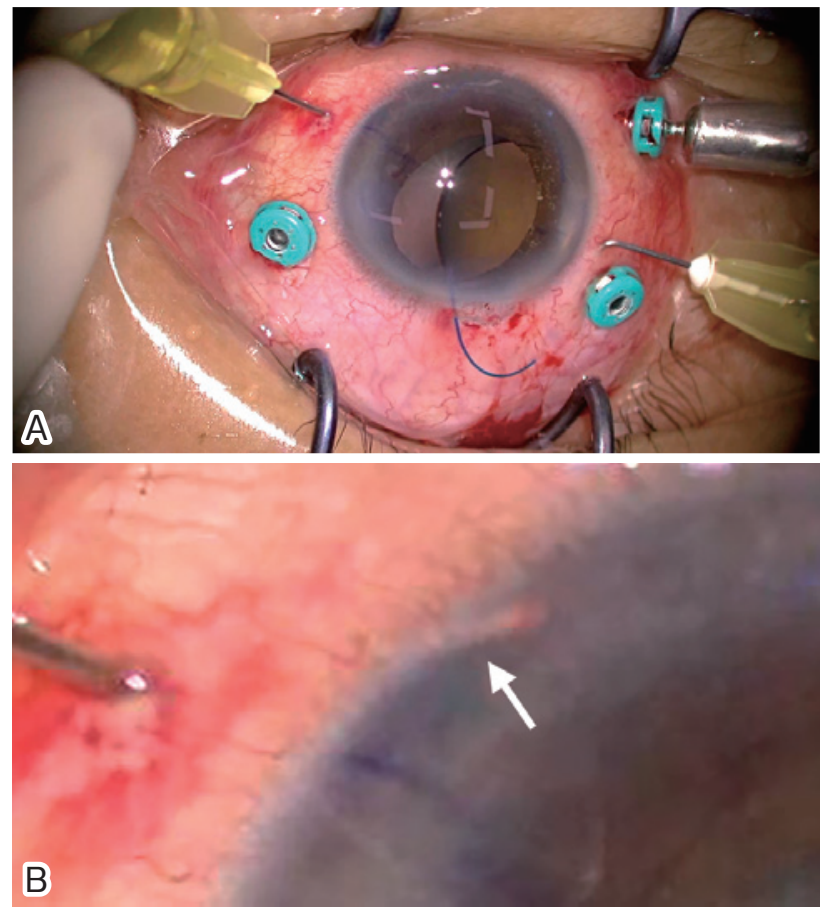

Fig. 2 Iris damage.

A) Iris damage from a $30-G$ needle.

B) Iris damage at higher magnification (arrow).

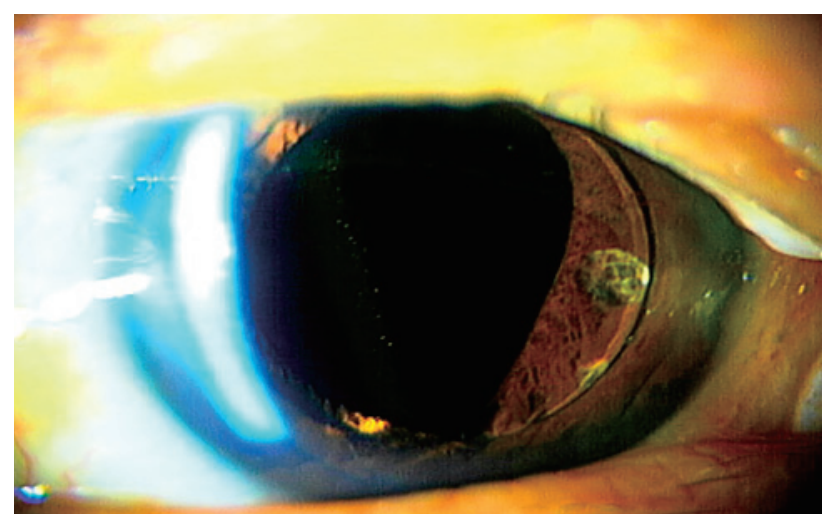

Fig. 3 Iris capture.

posterior chamber IOL in 1 case, subluxated cataract in 1 , and aphakic eyes in 2 in Group T, and dislocated posterior chamber IOL in 2 cases, subluxated cataract in 1, and aphakic eyes in 3 in Group F. Mean follow-up was $12.5 \pm$ 7.9 months in Group $\mathrm{T}$ and $14.1 \pm 2.1$ months in Group F. Four eyes underwent 25-G pars plana vitrectomy for indications such as dislocated IOL and subluxated cataract. Intraoperative complications included iris damage from the 30-G needle in 2 eyes (Group F; Fig. 2). Postoperative complications included iris capture by the IOL in 1 eye (Group F; Fig. 3). The position of the IOL was changed to the back of the iris with block sutures, which prevent forward movement of the IOL, by suturing with

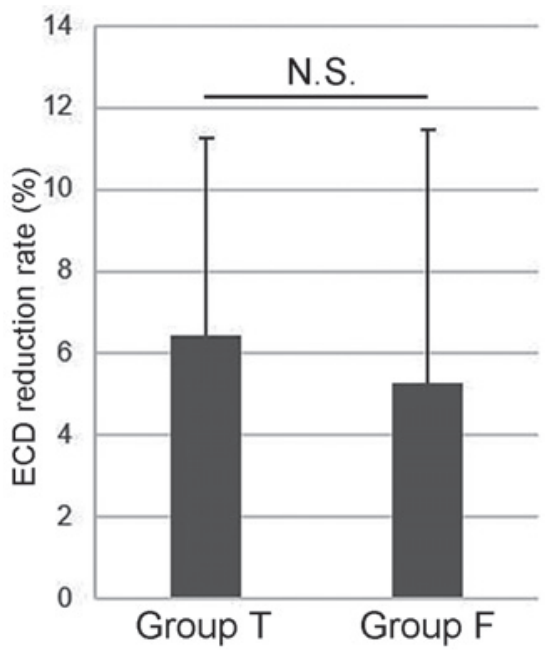

Fig. 4 Mean endothelial cell density reduction rate. ECD reduction rate was calculated from preoperative ECD to ECD at 3 months. Data were analyzed using the MannWhitney U-test. Values represent mean \pm standard deviation. N.S., not significant.

10-0 polypropylene between the iris and IOL. No postoperative retinal detachment or IOL dislocation developed.

Mean postoperative best-corrected visual acuity (BCVA) changed from -0.079 to $0.046 \log$ MAR at 3 months, after excluding 1 case with a final BCVA of 1.69 $\log$ MAR caused by proliferative diabetic retinopathy. The ECD reduction rate did not significantly differ between the 2 groups (Mann-Whitney U-test; Fig. 4). Next, we analyzed surgical videos from our database and determined the intraoperative times of steps related to intrascleral IOL fixation in both groups. Mean intraoperative times for intrascleral IOL fixation techniques were $27.5 \pm$ 9.7 min (Group T) and $13.0 \pm 3.8 \mathrm{~min}$ (Group F). Intraoperative time was significantly shorter for Group $\mathrm{F}$ than for Group T (P = 0.03, Mann-Whitney U-test) (Fig. 5).

\section{Discussion}

Ohta developed an intrascleral fixation technique that does not require large lamellar scleral flaps or fibrin glue $^{8,9}$. Yamane developed a surgical procedure that can be carried out via the conjunctiva, in which the haptics of the IOL are securely fixed to the sclera without using sutures or glue ${ }^{10,11}$. The current study was done to compare T-fixation and flanged IOL fixation in relation to intraoperative time (from IOL insertion to fixation) and complications in our initial cases. No IOL tilt or decentration was seen in any patient during follow-up. Both techniques yielded favorable surgical outcomes.

Intraoperative time was significantly shorter in Group $\mathrm{F}$ than in Group T, reflecting the fewer surgical proce- 


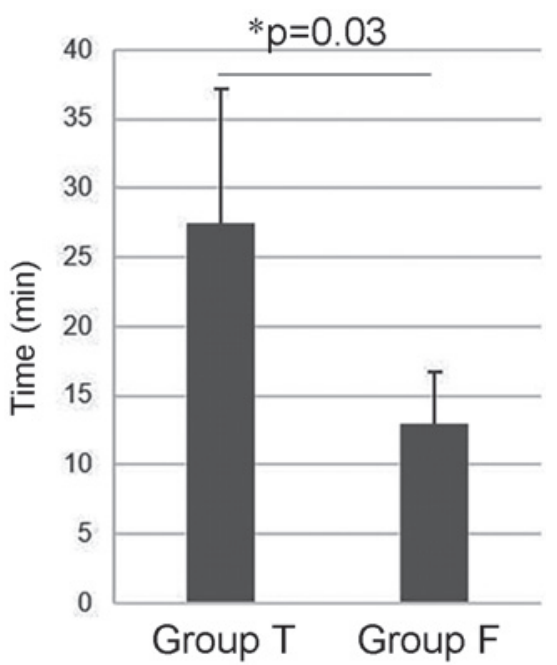

Fig. 5 Mean intraoperative time from IOL insertion to fixation.

Intraoperative time for steps related to intrascleral IOL fixation for T-fixation (Group T) and flanged IOL fixation (Group F). Data were analyzed with the Mann-Whitney U-test.

dures in Group F. However, no intra- or postoperative complications were seen in Group T, while intraoperative iris damage by the $30-\mathrm{G}$ needle and postoperative iris capture by the IOL were observed in Group F. With flanged IOL fixation, making an angled sclerotomy with a 30-G needle while avoiding iris injury is sometimes difficult, particularly in early cases. To avoid iris injury, we may use the Yamane double-needle stabilizer (GEUDER AG, Heidelberg, Germany), which helps simplify identification of sclerotomy sites and provides control over insertion angles. Needle insertion at a set angle, compared to the corneal limbus and iris, is easy with this device. Flanged IOL fixation may thus require a longer learning curve than T-fixation. The drawbacks of our study include its retrospective design, the fact that the results are from a single surgeon, the small number of cases, and the lack of randomization and masking.

In summary, both techniques achieved favorable surgical outcomes. Although flanged IOL fixation required less intraoperative time, this technique might be difficult for early cases and require a longer learning period than T-fixation.

Conflict of Interest: The authors declare no conflicts of interest.

\section{References}

1. Drolsum L. Long-term follow-up of secondary flexible, open-loop, anterior chamber intraocular lenses. J Cataract Refract Surg. 2003;29:498-503.

2. Biro Z. Results and complications of secondary intraocular lens implantation. J Cataract Refract Surg. 1993;19:647.

3. Downing JE. Ten-year follow up comparing anterior and posterior chamber intraocular lens implants. Ophthalmic Surg. 1992;23:308-15.

4. Lin CP, Tseng HY. Suture fixation technique for posterior chamber intraocular lenses. J Cataract Refract Surg. 2004; 30:1401-4.

5. Gabor SG, Pavlidis MM. Sutureless intrascleral posterior chamber intraocular lens fixation. J Cataract Refract Surg. 2007;33:1851-4.

6. Agarwal A, Kumar DA, Jacob S, Baid C, Agarwal A, Srinivasan S. Fibrin glue-assisted sutureless posterior chamber intraocular lens implantation in eyes with deficient posterior capsules. J Cataract Refract Surg. 2008;34: 1433-8.

7. Rodriguez-Agirretxe I, Acera-Osa A, Ubeda-Erviti M. Needle-guided intrascleral fixation of posterior chamber intraocular lens for aphakia correction. J Cataract Refract Surg. 2009;35:2051-3.

8. Ohta T, Toshida H, Murakami A. Simplified and safe method of sutureless intrascleral posterior chamber intraocular lens fixation: Y-fixation technique. J Cataract Refract Surg. 2014;40:2-7.

9. Ohta T. Kyomakunaikoteijutsu no subete [All of intrascleral fixation technique]: T-fixation technique. IOL \& RS. 2015;29:162-8. Japanese.

10. Yamane S, Inoue M, Arakawa A, Kadonosono K. Sutureless 27-gauge needle-guided intrascleral intraocular lens implantation with lamellar scleral dissection. Ophthalmology. 2014;121:61-6.

11. Yamane S, Sato S, Maruyama-Inoue M, Kadonosono K. Flanged intrascleral intraocular lens fixation with doubleneedle technique. Ophthalmology. 2017;124:1136-42.

12. Kelkar AS, Fogla R, Kelkar J, Kothari AA, Mehta H, Amoaku W. Sutureless 27-gauge needle-assisted transconjunctival intrascleral intraocular lens fixation: initial experience. Indian J Ophthalmol. 2017;65:1450-3.

13. Totan Y, Karadag R. Trocar-assisted sutureless intrascleral posterior chamber foldable intra-ocular lens fixation. Eye. 2012;26:788-91.

(Received, October 23, 2019)

(Accepted, November 22, 2019)

(J-STAGE Advance Publication, December 27, 2019)

Journal of Nippon Medical School has adopted the Creative Commons Attribution-NonCommercial-NoDerivatives 4.0 International License (https://creativecommons.org/licenses/by-nc-nd/4.0/) for this article. The Medical Association of Nippon Medical School remains the copyright holder of all articles. Anyone may download, reuse, copy, reprint, or distribute articles for non-profit purposes under this license, on condition that the authors of the articles are properly credited. 